\title{
Influence of benzodiazepines on antiparkinsonian drug treatment in levodopa users
}

van de Vijver DAMC, Roos RAC, Jansen PAF, Porsius AJ, de Boer A. Influence of benzodiazepines on antiparkinsonian drug treatment in levodopa users.

Acta Neurol Scand 2002: 105: 8-12. (C) Munksgaard 2002.

Objectives - Animal studies showed that benzodiazepines decrease the concentration of dopamine in the striatum. Benzodiazepines may therefore affect the treatment of Parkinson's disease. This study determined whether start of a benzodiazepine in patients on levodopa was followed by a faster increase of antiparkinsonian drug treatment. Methods - Data came from the PHARMO database, which includes information on drug dispensing for all residents of six Dutch cities. Selected were all patients aged 55 years and older who used levodopa for at least 360 days. The rate of increase of antiparkinsonian drug treatment was compared between starters of a benzodiazepine and controls who did not start a benzodiazepine with the use of Cox's proportional hazard model. Results - Identified were 45

benzodiazepine starters ( 27 women, mean age 76.4 years) and 169 controls (83 women, 74.3 years). Antiparkinsonian drug treatment increased faster in the benzodiazepine group; relative risk was 1.44 (95\% confidence interval 0.80-2.59). Conclusion - This study has not found any statistically significant increase in antiparkinsonian drug treatment when a benzodiazepine was started in a small population of chronic levodopa users.
D. A. M. C. van de Vijver ${ }^{1}$, R. A. C. Roos ${ }^{2}$, P. A. F. Jansen, A. J. Porsius' ${ }^{1}$, A. de Boer ${ }^{1}$

${ }^{1}$ Department of Pharmacoepidemiology and Pharmacotherapy, Utrecht Institute for Pharmaceutical Sciences (UIPS), Utrecht University, Utrecht, the Netherlands; ${ }^{2}$ Department of Neurology, Leiden University Medical Centre (LUMC), Leiden University, Leiden, the Netherlands; ${ }^{3}$ Department of Geriatric Medicine, University Medical Centre Utrecht (UMCU), Utrecht University, Utrecht, the Netherlands

Key words: Parkinson's disease; levodopa benzodiazepine; pharmacoepidemiology; antiparkinsonian drug treatment

David van de Vijver, Department of pharmaco-epidemiology and -therapy, Utrecht Institute for Pharmaceutical Sciences (UIPS), Utrecht University, PO Box 80 082, 3508 TB Utrecht, the Netherlands

Tel.: +31302537324

Fax: +31302539166

e-mail: d.a.m.c.vandevijver@pharm.uu.nl

Accepted for publication June 19, 2001
Loss of dopamine in the striatum as a result of neuronal degeneration of the substantia nigra pars compacta is a major characteristic in the pathology of Parkinson's disease. Animal studies have shown that $\gamma$-amino butyric acid $\mathrm{A}\left(\mathrm{GABA}_{\mathrm{A}}\right)$-agonists decrease extracellular concentrations of dopamine in the striatum (1-3). An important class of drugs that stimulate $\mathrm{GABA}_{\mathrm{A}}$-receptors are benzodiazepines (4). These drugs may therefore worsen the symptoms of Parkinson's disease. Patients with Parkinson's disease often use benzodiazepines (5), as sleep disorders and anxiety are common $(6,7)$.

Several studies reported on the effects of benzodiazepines on Parkinson's disease (8-12). These studies, which were all based on small numbers of patients, found conflicting results.

Dopaminergic effects, such as improvement of motorsymptoms (9), as well as antidopaminergic effects, such as worsening of Parkinson's disease and decrease of dyskinesia (8-10), have been reported. In some patients, benzodiazepines did not have any apparent effect $(9,11,12)$. As animal studies reported that stimulation of $\mathrm{GABA}_{\mathrm{A}}$ inhibits dopamine (1-3), it is expected that patients on levodopa who are given a benzodiazepine require adjustment of antiparkinsonian drug treatment. Such an adjustment can be studied in pharmacy records (13).

We used pharmacy records to determine whether start of a benzodiazepine in patients on levodopa was followed by a faster increase of antiparkinsonian drug treatment compared with levodopa users who did not start with benzodiazepines.

\section{Methods}

Data

Data were obtained from the PHARMO system, which includes information on drug dispensing for all 300,000 residents of six Dutch cities from 1991 
to 1998 (14). In the Netherlands virtually complete histories can be obtained for all patients. This is because of a strong pharmacy-patient liaison for reimbursement of prescription drugs, a high degree of computerization, the use of standardized computer software, classification and coding systems, and a strong commitment of pharmacists to medication surveillance. Antiparkinsonian drugs and benzodiazepines were fully covered by insurance from 1991 to 1998.

\section{Study population}

In a previous study, we compared pharmacy records with the results of the Rotterdam Study $(15,16)$. All participants of the Rotterdam Study, almost 7000 persons of 55 years and older, were examined for signs of Parkinson's disease with the use of internationally accepted diagnostic criteria (16). We found that $95 \%$ of the patients using levodopa had Parkinson's disease. Levodopa is therefore a reliable marker for Parkinson's disease in pharmacy records among individuals aged 55 years and older (15).

For the present study we selected all patients aged 55 years and older, who used levodopa for at least 360 days. From these patients, a group of starters of a benzodiazepine and a control group were formed. Patients were included in the benzodiazepine group when they had started using a benzodiazepine at least 180 days later than the first prescription of levodopa in PHARMO and continued using the benzodiazepine together with levodopa for at least another 180 days. All other levodopa users were allocated to the control group.

\section{Follow-up}

Start of follow-up in the benzodiazepine group was the first dispensing date of the benzodiazepine. In this group, patients started using a benzodiazepine at average 872 days later than the first prescription for levodopa in PHARMO. Start of follow-up in the control group was therefore taken at the first dispensing date of levodopa that was at least 872 days later than the start of levodopa in the file. Controls who subsequently used levodopa for less than 180 days were not included. Some patients had used a benzodiazepine but did not meet the inclusion criteria for the benzodiazepine group. These patients were only included as control when they had not used a benzodiazepine in the 180 days before start of follow-up.

A possible disadvantage of taking a fixed period of duration of levodopa among controls is that their little variation in duration of levodopa use in
PHARMO does not allow a valid comparison with benzodiazepine starters with a larger variation of duration of levodopa use. However, most patients started using levodopa before they entered PHARMO. Therefore, duration of levodopa use in PHARMO does not reflect duration of use of levodopa by a patient.

Excluded were patients who used antipsychotics, metoclopramide (17), cinnarizine and flunarizine (18) at start of follow-up. These agents can induce parkinsonism and are therefore potentially interfering with treatment of Parkinson's disease. Furthermore, users of selective serotonin reuptake inhibitors (SSRIs) and tricyclic antidepressants were excluded, because they are associated with, respectively, a higher and a lower rate of increase of antiparkinsonian drug treatment in levodopa users (13). Omitted were patients who used an antiepileptic drug at start of follow-up, because some of these drugs act through stimulation of GABA (19).

The endpoint of follow-up was reached if an increase of antiparkinsonian drug treatment occurred within 180 days after start of follow-up. This increase occurred when the dosage of any antiparkinsonian drug was increased, or a new antiparkinsonian drug was started. The antiparkinsonian drugs in our study next to levodopa were: dopamine agonists (bromocriptine, lisuride, pergolide, pramipexole, ropinirole), selegiline, amantadine, anticholinergics (biperidene, dexetimide, orphenadrine, procyclidine, trihexifenidyl), and the catechol O-methyl transferase (COMT) inhibitors entacapone and tolcapone. A patient was censored when no increase in antiparkinsonian drug treatment occurred within 180 days. Patients were also censored when an antipsychotic, metoclopramide, cinnarizine or flunarizine, a tricyclic antidepressant, a SSRI, or an antiepileptic drug was started during follow-up.

A sample size calculation was performed. In a previous study we found that $25 \%$ of chronic levodopa users received a benzodiazepine (5). We assumed that a relative risk of 2.0 is relevant. Sample size calculation (20) showed that we needed to include at least 31 benzodiazepine starters and 93 controls to detect such a relative risk in our study with $80 \%$ power, given a type 1 probability of 0.05 .

\section{Analysis}

The rate of increase of antiparkinsonian drug treatment was compared between the benzodiazepine and control group with Kaplan-Meier survival curves. The relative risk and 95\% 
confidence intervals were calculated using Cox's proportional hazard model (21), which enabled adjustment for age, gender, calendar year, and the time a patient had received levodopa in the file before start of follow-up. We adjusted for calendar-year to control for possible changes in prescription patterns.

\section{Results}

A total of 662 patients aged 55 years and older used levodopa for at least 360 days. Of these patients, 52 started a benzodiazepine at least 180 days after start of levodopa in the file and used the benzodiazepine together with levodopa for another 180 days. Five benzodiazepine starters were excluded, because they used an antipsychotic at start of follow-up. Two patients were excluded because they used a tricyclic antidepressant. The remaining 45 patients used the following benzodiazepines: diazepam $(n=3)$, chlordiazepoxide $(n=3)$, oxazepam $(n=10)$, flurazepam $(n=2)$, nitrazepam $(n=4)$, lormetazepam $(n=3)$, and temazepam $(n=20)$.

Identified were 330 patients who fulfilled our criteria for being included as a control, i.e. they filled a prescription for levodopa at least 872 days after the first dispensing date for levodopa in PHARMO, and subsequently used levodopa for at least another 180 days. From these 330 controls we excluded 50 patients because they used at least one of the following potentially interfering drugs at start of follow-up: antipsychotics $(n=9)$, cinnarizine or flunarizine $(n=7)$, metoclopramide $(n=2)$, a tricyclic antidepressant $(n=19)$, or a SSRI $(n=19)$. (Adding up the number between parentheses exceeds 50 because some patients used two or more of the drugs listed.) No patient used an antiepileptic drug at start of follow-up. A number of 111 patients were excluded as control, because they used a benzodiazepine during or in the 180 days before start of follow-up. This left 169 patients who were included as control.

Table 1 lists the characteristics of the 45 benzodiazepine starters and the 169 controls. The proportion of female patients was highest among benzodiazepine users. Mean levodopa dosage and the distribution of antiparkinsonian drug users were comparable between the groups.

Table 2 shows the endpoints of the different groups. The proportion of persons who did not experience an increase of antiparkinsonian drug treatment was slightly higher among controls.

Antiparkinsonian drug treatment increased faster among levodopa users who used a benzo-
Table 1. Baseline characteristics of benzodiazepine users and their controls

\begin{tabular}{lcc}
\hline Characteristic & Benzodiazepine & Controls \\
\hline Number & 45 & 169 \\
Female & $27(60)$ & $83(49)$ \\
Mean age (years) \pm SD & $76.4 \pm 7.1$ & $74.3 \pm 7.8$ \\
Mean time on levodopa (days) \pm SD & $871 \pm 600$ & $911 \pm 47$ \\
Mean levodopa dosage \pm SD & $351 \pm 222$ & $340 \pm 191$ \\
Dopamine agonist & $10(22)$ & $34(20)$ \\
Selegiline & $16(36)$ & $67(40)$ \\
Amantadine & $5(11)$ & $14(8)$ \\
Anticholinergics & $5(11)$ & $34(20)$ \\
Start of follow-up & & \\
$\quad$ Before 1996 & $31(69)$ & $121(72)$ \\
1996 or later & $14(31)$ & $48(28)$ \\
\hline
\end{tabular}

Values in parentheses are in percentages.

* Entacapone and tolcapone were not used.

Table 2. Events at endpoint of follow-up for patients on levodopa who started using a benzodiazepine $(n=45)$, and controls $(n=169)$

\begin{tabular}{lcc}
\hline Endpoint & $\begin{array}{c}\text { Benzodiazepine } \\
(n=45)\end{array}$ & $\begin{array}{c}\text { Controls } \\
(n=169)\end{array}$ \\
\hline Increase of antiparkinsonian drug treatment & $7(16)$ & $23(14)$ \\
Levodopa & $2(4)$ & $10(6)$ \\
Dopamine agonist & $4(9)$ & $7(4)$ \\
Selegiline & $1(2)$ & $4(2)$ \\
Amantadine & $1(2)$ & $3(2)$ \\
Anticholinergic & & \\
Censoring* & $30(67)$ & $121(72)$ \\
$\quad$ No change of antiparkinsonian drug treatment & 0 & $1(1)$ \\
Start of antipsychotic & & \\
\hline
\end{tabular}

Values in parentheses are in percentages.

* Censoring because of start of cinnarizine, flunarizine or metoclopramide did not occur.

diazepine (Fig. 1). The crude relative risk comparing both groups was 1.29 (95\% confidence interval $0.72-2.31$ ). Adjusting for age, gender and time on levodopa, the relative risk increased to $1.44(0.80-$ 2.72).

\section{Discussion}

This study has not found any statistically significant increase in antiparkinsonian drug treatment when a benzodiazepine was started in a small population of chronic levodopa users.

It should be acknowledged that this study is a mere indication that use of benzodiazepines by patients with Parkinson's disease will lead to only a slight increase of antiparkinsonian drug treatment. A definite answer to this issue can only be given after clinical assessment of the patients. We did not have access to clinical data.

We assessed in a previous study that levodopa is a reliable marker for Parkinson's disease (15). We therefore think that we did not include too many 


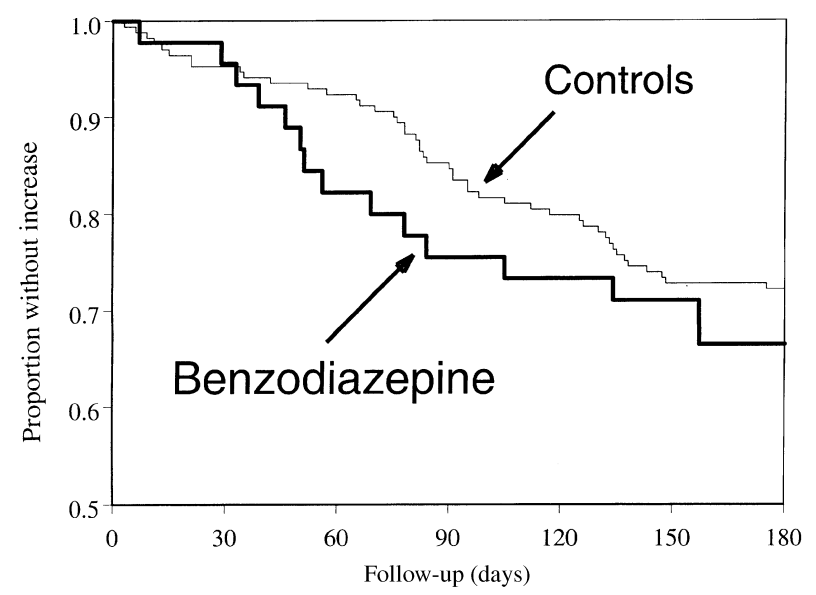

Fig. 1. Comparison of the proportion of patients without increase of antiparkinsonian drug treatment (Kaplan-Meier estimate) within 180 days after start of follow-up.

patients who had a parkinsonian syndrome other than Parkinson's disease. It should be stated that postmortem examination has shown that $25 \%$ of all patients were incorrectly diagnosed with Parkinson's disease (22).

We do not think that our study is hampered by noncompliance. Both the benzodiazepine starters and the controls are comparable with respect to use of antiparkinsonian drugs. The proportion of patients who are not compliant, will therefore be the same in both groups. Furthermore, use of benzodiazepines for at least 180 days causes dependence. The benzodiazepine users are therefore expected to be compliant.

The fact that we did not find a statistically significant increase of antiparkinsonian drug treatment can be because of the absence of such an effect in humans. However, the lack of an effect can also be explained by several other reasons. First, benzodiazepines can be given for treatment of sleep disorders. Motorsymptoms in Parkinson's disease may benefit from sleep, allowing patients to delay their antiparkinsonian medication in the morning (23-25). This may influence the results of this study. It should be noted that this beneficial effect has not been determined for sleep induced by benzodiazepines.

A second reason for finding only a slight increase is that benzodiazepine may inhibit dopamine release in a dose dependent manner (26). Dosages in our study may have been too low to find an effect. In animal studies dosages were as high as $1 \mathrm{mg} / \mathrm{kg}$ bodyweight (2), which is far higher than used in clinical practice (4). Our data did not allow to study a dose-relation, because the majority of patients used the same dose of a specific benzodiazepine.
The third reason is that benzodiazepines reduce the psychological burden of the disease. Patients can therefore be able to cope better with their disease, which can be beneficial for motor performance.

Other studies have reported conflicting results on the effects of benzodiazepines in Parkinson's disease (8-12). The dissimilarities in the results can be because of methodological differences. Pourcher et al. (8), who found that diazepam reduced levodopa-induced dyskinesia, administered the benzodiazepine intramuscular. Furthermore, two studies, that reported both a detrimental and an absence of an effect of benzodiazepines, were case reports $(9,10)$. The patients in these case reports were described for their exceptional reaction towards benzodiazepines and may thus not be representative for effects normally encountered in Parkinson's disease. The result of one study, that found no apparent effect, was based on a single dose of temazepam (11). This may have been too short to observe any effect.

Recently two papers have been published that described the beneficial effects of zolpidem on Parkinson's disease $(27,28)$. Zolpidem is a nonbenzodiazepine hypnotic, which shows a preferential binding to a specific $\mathrm{GABA}_{\mathrm{A}}$ receptor subtype (4). In our study no patients were identified that used zolpidem for a sufficient time.

The hypothesis that benzodiazepines may be associated with a higher rate of increase of antiparkinsonian drug treatment is based on animal studies which showed that stimulation of $\mathrm{GABA}_{\mathrm{A}}$ decreases the activity of dopamine in the striatum (1-3). The relation between $\mathrm{GABA}_{\mathrm{A}}$ and dopamine in the striatum is based on several observations. First, $\mathrm{GABA}_{\mathrm{A}}$-projections inhibit dopaminergic neurones in the substantia nigra in vivo (1). Secondly, clonazepam decreased turning behaviour that was induced by the potent dopamine agonist apomorphine. This antidopaminergic action of clonazepam was reduced by pretreatment with the benzodiazepine antagonist flumazenil (2). Finally, the striatal concentration of dopamine was increased by $\mathrm{GABA}_{\mathrm{A}}$-antagonists and decreased by $\mathrm{GABA}_{\mathrm{A}}$-agonists (3).

Our study shows that benzodiazepines are not associated with an increase of antiparkinsonian drug treatment in a population in daily practice. Benzodiazepines should always be used cautiously in Parkinson's disease, because benzodiazepines are associated with an increased frequency of femour fractures (29). Patients with Parkinson's disease are likely to fall because of postural stability (6).

This study shows that benzodiazepines are associated with a slight increase of antiparkinsonian 
drug treatment among levodopa users. However, this increase is not statistically significant. The benzodiazepine induced striatal decrease of dopaminergic concentrations observed in animals, does not seem to have a relevant effect on Parkinson's disease in humans.

\section{Acknowledgements}

This study was supported financially by the royal Dutch organization for the advancement of pharmacy (KNMP) and Utrecht Institute for Pharmaceutical Sciences (UIPS) of Utrecht University.

\section{References}

1. Paladini CA, Celada P, Tepper JM. Striatal, pallidal, and pars reticulata evoked inhibition of nigrostriatal dopaminergic neurons is mediated by GABA (A) receptors in vivo. Neuroscience 1999;89:799-812.

2. TenN CC, Niles LP. Central-type benzodiazepine receptors mediate the antidopaminergic effect of clonazepam and melatonin in 6-hydroxydopamine lesioned rats: involvement of a GABAergic mechanism. J Pharmacol Exp Ther 1995;274:84-9.

3. Smolders i, De Klippel N, Sarre S, Ebinger G, MichOTTE Y. Tonic GABA-ergic modulation of striatal dopamine release studied by in vivo microdialysis in the freely moving rat. Eur J Pharmacol 1995;284:83-91.

4. Hobbs WT, Rall T, Verdoorn T. Hypnotics and sedatives; ethanol. In: Hardman J, Limbird L, Molinoff P, Ruddon R, Goodman Gilman A, eds. Goodman and Gilman's the pharmacological basis of therapeutics. New York: McGraw-Hill, 1996:361-96.

5. van de Vijver DAMC, de Boer A, Herings RMC, Roos RAC, Porsius AJ. Increased use of psychotropic drugs by patients with Parkinson's disease. Br J Clin Pharmacol 1999;47:476P.

6. Olanow CW, Koller WC. An algorithm (decision tree) for the management of Parkinson's disease: treatment guidelines. Am Acad Neurol Neurol 1998;50:S1-57.

7. Aarsland D, Larsen JP, Lim NG et al. Range of neuropsychiatric disturbances in patients with Parkinson's disease. J Neurol Neurosurg Psychiatry 1999;67:492-6.

8. Pourcher E, Bonnet AM, Kefalos J, Dubois B, Agid Y. Effects of etybenzatropine and diazepam on levodopainduced diphasic dyskinesias in Parkinson's disease. Mov Disord 1989;4:195-201.

9. Hunter KR, Stern GM, Laurence DR. Use of levodopa with other drugs. Lancet 1970;2:1283-5.

10. Yosselson-Superstine S, Lipman AG. Chlordiazepoxide interaction with levodopa. Ann Intern Med 1982;96: 259-60.

11. Tulloch JA, Ashwood TJ, Bateman DN, Woodhouse $\mathrm{KW}$. A single-dose study of the pharmacodynamic effects of chlormethiazole, temazepam and placebo in elderly parkinsonian patients. Age Ageing 1991;20:424-9.

12. Koller WC, Herbster G. Adjuvant therapy of parkinsonian tremor. Arch Neurol 1987;44:921-3.
13. van de Vijver DAMC, Roos RAC, JAnsen PAF, Porsius AJ, DE Boer A. Start of a selective serotonin reuptake inhibitor (SSRI) and increase of antiparkinsonian drug treatment in patients on levodopa. Pharmacoepidemiol Drug Saf 2000;9:S11-S12.

14. Herings RM, Bakker A, Stricker BH, Nap G. Pharmaco-morbidity linkage: a feasibility study comparing morbidity in two pharmacy based exposure cohorts. J Epidemiol Community Health 1992;46:136-40.

15. van de Vijver DAMC, Stricker BHC, Breteler MMB, Roos RAC, Porsius AJ, DE Boer A. Evaluation of antiparkinsonian drugs in pharmacy records as a marker for Parkinson's disease. Pharm World Sci 2001;23:148-52.

16. De Rijk MC, Breteler MMB, Graveland GA et al. Prevalence of Parkinson's disease in the elderly: the Rotterdam study. Neurology 1995;45:2143-6.

17. Miller LG, Jankovic J. Metoclopramide-induced movement disorders. Clinical findings with a review of the literature. Arch Intern Med 1989;149:2486-92.

18. Negrotti A, Calzetti S. A long-term follow-up study of cinnarizine- and flunarizine-induced parkinsonism. Mov Disord 1997;12:107-10.

19. McNamara J. Drugs effective in the therapy of the epilepsies. In: Hardman J, Limbird L, Molinoff P, Ruddon R, Goodman Gilman A, eds. Goodman and Gilman's the pharmacological basis of therapeutics. New York: McGraw-Hill, 1996:461-86.

20. Schoenfeld DA, Richter JR. Nomograms for calculating the number of patients needed for a clinical trial with survival as an endpoint. Biometrics 1982;38:163-70.

21. Hosmer D, Lemeshow S. Applied survival analysis. Regression modeling of time to event data. New York: John Wiley, 1999:386.

22. Hughes AJ, Daniel SE, Kilford L, Lees AJ. Accuracy of diagnosis of idiopathic Parkinson's disease: a clinicopathological study of 100 cases. J Neurol Neurosurg Psychiatry 1992;55:181-4.

23. Bateman DE, Levett K, Marsden CD. Sleep benefit in Parkinson's disease. J Neurol Neurosurg Psychiatry 1999;67:384-5.

24. Merello M, Hughes A, Colosimo C, Hoffman M, Starkstein S, Leiguarda R. Sleep benefit in Parkinson's disease. Mov Disord 1997;12:506-8.

25. TANDberg E, LARSEn JP, Karlsen K. Excessive daytime sleepiness and sleep benefit in Parkinson's disease: a community-based study. Mov Disord 1999;14:922-7.

26. Takada K, Murai T, Kanayama T, Koshikawa N. Effects of midazolam and flunitrazepam on the release of dopamine from rat striatum measured by in vivo microdialysis. Br J Anaesth 1993;70:181-5.

27. Ruzicka E, Roth J, Jech R, Busek P. Subhypnotic doses of zolpidem oppose dopaminergic-induced dyskinesia in Parkinson's disease. Mov Disord 2000;15:734-5.

28. Daniele A, Albanese A, Gainotti G, Gregori B, Bartolomeo P. Zolpidem in Parkinson's disease. Lancet 1997;349:1222-3.

29. Herings RM, Stricker BH, de Boer A, Bakker A, Sturmans F. Benzodiazepines and the risk of falling leading to femur fractures. Dosage more important than elimination half-life. Arch Intern Med 1995;155: 1801-7. 